\title{
The Power of the KPSS-Test for Cointegration when Residuals are Fractionally Integrated
}

\author{
Philipp Sibbertsen ${ }^{1}$ \\ Walter Krämer ${ }^{2}$ \\ Diskussionspapier 318 \\ ISNN 0949-9962
}

\begin{abstract}
We show that the power of the KPSS-test against integration, as measured by divergence rates of the test statistic under the alternative, remains the same when residuals from an OLSregression rather than true observations are used. The divergence rate is independent of the order of integration of the cointegrating regressors which are allowed to be $I\left(1+d_{X}\right)$ in our set up.
\end{abstract}

KEYwORDS: Keywords: cointegration, power, long memory, KPSS-Test.

JEL-Classification: C 12; C 32

\footnotetext{
${ }^{1}$ Universität Hannover, Fakultät Wirtschaftswissenschaften, Abteilung Statistik Königsworther Platz 1, 30167 Hannover

Fax-Nr. +49- 511- 7623923

E-Mail: sibbertsen@statistik.uni-hannover.de

${ }^{2}$ Universität Dortmund, Fachbereich Statistik

44221 Dortmund

E-Mail: walterk@statistik.uni-dortmund.de
} 


\section{Introduction and Summary}

This paper is concerned with the Kwiatkowski-Phillips-Schmidt-Shin (1992, KPSS) procedure for testing the null hypothesis that there is at least one cointegrating relationship among $m I(1)$ variables $z_{t 1}, \ldots, z_{t m}$. If all potential cointegrating relationships are known, this simply amounts to applying the standard KPSS-test to these relationships. If a cointegrating relationship has to be estimated, one applies the KPSS-test to the residuals from this regression.

Lee and Schmidt (1996) show that the KPSS-test statistic diverges when the residuals from the cointegrating relationship are $I(d)$-processes with $-0.5<$ $d<0.5$; Marmol (1997) shows that the KPSS-test is also consistent against $I(d)$-processes with $d \geq 0.5$. However, both Lee and Schmidt (1996) and Marmol (1997) assume that the true cointegrating relationship is known.

These authors assume in addition that the cointegrating variables $z_{t 1}, \ldots, z_{t m}$ are $I(1)$. We extend this approach by allowing them to be fractionally integrated i. e. to be $I\left(1+d_{X}\right)$ where $-1 / 2<d_{X}<1 / 2$ denotes the order of integration of the cointegrating regressor. Therefore, we relax the standard 'I(1)/I(0)' approach in cointegration not only for the residuals but also for the cointegrating regressor. Practical experience shows that the classical approach is often too restrictive as many time series show a behaviour which is somewhat between $I(1)$ and $I(0)$ such as stationary long memory or non-stationary but still mean-reverting time series. This wide range of persistence characteristic is parsimoniously captured by $I(d)$ processes where $d$ can take any value between $-1 / 2$ and 1 . The value $d=1 / 2$ characterizes the border between stationarity and non-stationarity.

The case where the cointegrating relationship has to be estimated has been considered in great detail by Phillips and Ouliaris (1990), but only for tests of the complementary null hypothesis that there is no cointegration. The behaviour of tests for this null hypothesis when the residuals are fractionally integrated has been considered by Krämer and Marmol (2004) but again under the assumption of $I(1)$ cointegrating regressors. Below we consider the 
null hypothesis that cointegration does exist. It emerges that the divergence rates under the alternatives from Lee and Schmidt (1996) and Marmol (1997) remain the same. We also prove that the divergence rate does not depend on the order of the cointegrating regressors. This is a rather surprising result.

Our approach extends previous results in two directions. First, we allow the cointegrating regressors to be fractionally integrated. This extends especially the results of Krämer and Marmol (2004). Second, we consider the KPSSprocedure when the cointegrating relationship is unknown and has to be estimated which extends the previous results concerning the KPSS-test by Marmol (1997) and Lee and Schmidt (1996).

\section{Power under the alternative}

Other than Krämer and Marmol (2004), Marmol (1997) and Lee and Schmidt (1996) who assume the cointegrating regressors to be $I(1)$ we consider the regression model

$$
y_{t}=\beta^{\prime} z_{t}+u_{t},
$$

where the m-vector $z_{t}$ is defined by $z_{t}=\sum_{s=1}^{t} x_{s}$ and $x_{s} \sim I\left(d_{X}\right)$. This implies that $z_{t} \sim I\left(1+d_{X}\right)$ and that all elements of the m-vector of regressors are integrated to the same order. We then postulate that $y_{t} \sim I\left(1+d_{X}\right)$, but that $u_{t} \sim I\left(d_{u}\right)$ where $-1 / 2<d_{u}<1 / 2<1+d_{X}$, implying what is called fractional cointegration. We consider the null hypothesis that there is exactly one cointegrating relationship.

The KPSS-procedure for testing this hypothesis is based on the statistic

$$
\hat{\eta}=\frac{1}{T^{2}} \sum_{t=1}^{T} S_{t}^{2} / s^{2}(\ell),
$$

where

$$
S_{t}=\sum_{i=1}^{t} u_{i}-\bar{u},
$$




$$
s^{2}(\ell)=\frac{1}{T} \sum_{t=1}^{T}\left(u_{t}-\bar{u}\right)^{2}+\frac{2}{T} \sum_{s=1}^{\ell} w_{s \ell} \sum_{t=s+1}^{T}\left(u_{t}-\bar{u}\right)\left(u_{t-s}-\bar{u}\right)
$$

and where $w_{s \ell}=1-\frac{s}{\ell+1} s^{2}(\ell)$ is the Newey-West estimator of the long-run variance of the residuals. Its consistency in our long-memory set up follows from Hosking (1996) for $\ell=0$. Therefore, we restrict our attention to the case $\ell=0$ and disregard the estimation of the variance when computing the rates of divergence below.

If the $u_{t}$ 's are known and $u_{t} \sim I(0)$, we have

$$
\hat{\eta} \stackrel{d}{\longrightarrow} \int_{0}^{1} V(r)^{2} d r
$$

where $V(r)$ is a standard Brownian Bridge (see Kwiatkowski et al. 1992, or Lee and Schmidt 1996 for details). We consider the limiting distribution of $\hat{\eta}$ when the residuals $u_{t}$ in $(5)$ are $I(d)$ with $0<d_{u}<1$. For the case where the $u_{t}$ 's are known, and $0<d_{u}<0.5$, Lee and Schmidt (1996, Theorem 3) show that

$$
\hat{\eta}_{t}=O_{p}(T)^{2 d_{u}}
$$

For the case where $d_{u} \geq 0.5$, Marmol (1997) shows that

$$
\hat{\eta}_{t}=O_{p}(T)
$$

so that the KPSS-test is consistent against both stationary and nonstationary long memory alternatives.

So far, however, it has remained an open problem whether the above divergence rates carry over to the case where the $u_{t}$ 's are replaced by OLS-residuals. We consider this case in what follows by computing the divergence rate of the KPSS-test under fractional alternatives and allowing the regressors to be likewise fractionally integrated. This extends the usual situation of $I(0)$ regressors. But it can be shown that the structure of the regressors has no influence on 
the divergence rate of the test statistic.

THEOREM: Assume that $x_{t} \sim I\left(d_{X}\right),-\frac{1}{2}<d_{X}<\frac{1}{2}$ and $u_{t} \sim I\left(d_{u}\right), 0<$ $d_{u}<1+d_{X}$ and that the regressors are strictly exogenous. Then the rate of divergence of the KPSS-test is of order $O_{p}\left(T^{2 d_{u}}\right)$ irrespective of $d_{X}$.

REMARK: In the case of endogenous regressors we have to assume also $d_{X}+d_{u}>0$ to obtain the result. In the case of $d_{X}+d_{u} \leq 0$ the rate of convergence of $\hat{\beta}$ is of order $O_{p}\left(T^{1+2 d_{X}}\right)$ and therefore the considerations below are no longer valid. For details see Davidson (2004).

PROOF: We have:

$$
\hat{u}=u-Z^{\prime}\left(Z^{\prime} Z\right)^{-1} Z^{\prime} u \text {. }
$$

From Davidson (2004) we obtain for the least squares estimator of the cointegration parameter

$$
T^{1+d_{X}-d_{u}}(\hat{\beta}-\beta) \stackrel{d}{\longrightarrow} M
$$

with a matrix $M$ which is specified in Davidson (2004). In addition we obtain from Marinucci and Robinson (2000) that

$$
T^{-3 / 2-d_{X}} \sum_{t=1}^{[\lambda T]} z_{t} \stackrel{d}{\longrightarrow} B_{d}(\lambda),
$$

where $B_{d}(\lambda)$ denotes fractional Brownian motion with parameter $d=d_{X}+1$. Using the relation

$$
\hat{u}_{t}=u_{t}-(\hat{\beta}-\beta) z_{t}
$$

we obtain that $T^{-1 / 2-d_{u}} \sum_{t=1}^{[\lambda T]} u_{t}$ converges in distribution to some random variable, say $\xi(\lambda)$. The rate of convergence follows from (9), (10) and the 
application of Slutsky's Theorem to (11). The rate of convergence for the test statistic follows from the continuous mapping theorem, applying the same arguments as in Lee and Schmidt (1996).

REMARK: The limiting distribution in this case is not Gaussian.

The theorem shows that under the alternative of fractionally integrated cointegration residuals we obtain divergence rates independent of the structure of the regressors themselves. Furthermore, the limiting distribution is nonGaussian. The KPSS-test is thus powerful against fractional alternatives even when the cointegrating relationship has to be estimated and whether the regressors themselves have long memory or not.

\section{References}

Davidson, J. (2004) : "Convergence to stochastic integrals with fractionally integrated integrator processes: Theory and applications to cointegrating regression." Working Paper, Cardiff Business School, Cardiff.

Hosking, J. (1996) : "Asymptotic distribution of the sample mean, autocovariances and autocorrelations of long-memory time series." Journal of Econometrics $73,261-284$.

Krämer, W. and Marmol, F. (2004): "The power of residual-based tests for cointegration when residuals are fractionally integrated." Economics Letters 82, $63-69$.

Kwiatkowski, D.; Phillips, P.C.B.; Schmidt, P. and Shin, Y. (1992): "Testing the null hypothesis of stationarity against the alternative of a unit root: How sure are we that economic time series have a unit root?" Journal of Econometrics 54, 159 - 178. 
Lee, D. and Schmidt, P. (1996): "On the power of the KPSS test of stationarity against fractionally integrated alternatives." Journal of Econometrics $73,285-302$.

Marinucci, D. and Robinson, P. M. (2000): "Weak convergence of multivariate fractional processes." Stochastic Processes and their applications $86,103-120$.

Marmol, F. (1997): "Searching for fractional evidence using unit root tests." Mimeo, Universidad Carlos III, Madrid.

Phillips, P.C.B. and Ouliaris, S. (1990): "Asymptotic

properties of residual based tests for cointegration." Econometrica 58, $165-193$.

Phillips, P.C.B. and Perron, P. (1988) : "Testing for a unit root in time series regression." Biometrika 75, 335 - 346 . 